\title{
Obesidad y excreción urinaria de sodio en niños y adolescentes
}

\author{
ALICIA MASKIN L. ${ }^{1}$, MIRYAN LÓPEZ S. ${ }^{2}$, CLAUDIA MIR B. ${ }^{3}$, MARÍA ESTHER IBÁÑEZ P. $^{4}$
}

1. Bioquímica Especialista en Emergentología. Facultad de Ciencias Exactas, Químicas y Naturales; Universidad Nacional de Misiones (U.Na.M.), Posadas, Misiones, Argentina.

2. Bioquímica Especialista en Química Clínica. Facultad de Ciencias Exactas, Químicas y Naturales; Universidad Nacional de Misiones (U.Na.M.). Hospital Provincial de Pediatría Dr. Fernando Barreyro, Posadas, Misiones, Argentina.

3. Bioquímica. Facultad de Ciencias Exactas, Químicas y Naturales; Universidad Nacional de Misiones (U.Na.M.). Centro de Atención Primaria de la Salud No 17, Posadas, Misiones, Argentina.

4. Médica Pediatra Especialista en Nutrición. Hospital Provincial de Pediatría Dr. Fernando Barreyro, Posadas, Misiones, Argentina.

\begin{abstract}
Obesity and urinary sodium excretion in children and adolescents

Background: Obesity is a disease characterized by an abnormal accumulation body fat that results in neuroendocrine changes that alter the homeostasis of sodium, producing an increased risk of hypertension in adulthood. Objective: To evaluate the effect of obesity on urinary sodium excretion in children and adolescents. Subjects and Methods: 266 male and female children in the age group of 5-15 years were studied: 154 obese $(\mathrm{OB})$ with $\geq 95$ th percentile of BMI, and 112 normal-weight patients (C) with percentile 5-85 of BMI, from the outpatient service of the Children Hospital, Posadas, Misiones, Argentina during the years 2008 -2009. The determinations of serum sodium $\left(\mathrm{Na}_{\mathrm{s}}\right)$ and 24-hour urine $\left(\mathrm{Na}_{\text {ur }}\right)$ were performed using Ion Selective Electrode. The fractional excretion of sodium (FENa \%) was calculated. Results: $\mathrm{Na}_{\text {ur }}$ values (mEq/kg/day) and FENa \% reported significantly lower differences in the obese group compared to controls: $\mathrm{OB} \mathrm{Na}$ ur 2.23 vs $\mathrm{C} \mathrm{Na}_{\text {ur }} 3.40$ $(\mathrm{p}<0.0001)$; OB FENa \% 0.59 vs C FENa \% $0.71(\mathrm{p}=0.001)$. Conclusion: Results obtained in the present study showed that obese children have a significantly decreased urinary sodium excretion compared to normal weight children. This difference could be caused by renal retention of this ion.

(Key words: Obesity, natriuresis, urinary sodium, fractional excretion of sodium).

Rev Chil Pediatr 2012; 83 (5): 438-444
\end{abstract}

\section{RESUMEN}

Introducción: La obesidad es una enfermedad caracterizada por el aumento de grasa corporal, que genera modificaciones neuroendocrinas involucrando alteraciones en la homeostasis del sodio, que podrían generar hipertensión arterial en la adultez. Objetivo: Evaluar el efecto de la obesidad sobre la excreción urinaria de so-

Financiamiento: Facultad de Ciencias Exactas, Químicas y Naturales, UNaM, Misiones, Argentina.

Recibido el 13 de junio de 2011, devuelto para corregir el 12 de marzo de 2012, segunda versión el 31 de marzo de 2012 , tercera versión el 23 de abril de 2012, aceptado para publicación el 07 de mayo de 2012.

Este trabajo cumple con los requisitos sobre consentimiento /asentimiento informado, comité de ética, financiamiento, estudios animales y sobre la ausencia de conflictos de intereses según corresponda.

Correspondencia a:

Alicia M de Jensen.

E-mail: anoemijensen@hotmail.com 
dio en niños y adolescentes. Pacientes y Método: Se estudiaron 266 niños de ambos sexos, entre 5 y 15 años: 154 obesos (OB) definidos como IMC percentil $\geq 95$, y 112 normopeso (C), IMC percentil 5-85, provenientes del Servicio de Consultorio Externo del Hospital de Pediatría-Posadas, Misiones, Argentina, durante los años 2008-2009. Las determinaciones de sodio en suero $\left(\mathrm{Na}_{\mathrm{s}}\right)$ y orina de $24 \mathrm{~h}\left(\mathrm{Na}_{\mathrm{ur}}\right)$ se realizaron con electrodo ion selectivo. Se calculó la excreción fraccional de sodio (EFNa \%). Resultados: Los valores de $\mathrm{Na}_{\mathrm{ur}}(\mathrm{mEq} /$ $\mathrm{kg}$ /día) y la EFNa \% significativamente menores en el grupo de obesos con respecto a los controles: $\mathrm{Na}_{\text {ur }} \mathrm{OB}$ 2,23 vs $\mathrm{Na}_{\mathrm{ur}} \mathrm{C} 3,40(\mathrm{p}<0,0001)$; EFNa\% OB 0,59 vs EFNa \% C 0,71 (p=0,001). Conclusión: En el presente estudio los resultados mostraron que los niños y adolescentes obesos presentan una disminución significativa de la excreción urinaria de sodio respecto de los niños normopeso. Dicha diferencia podría estar generada por la retención renal de dicho ion.

(Palabras clave: Obesidad, natriuresis, sodio urinario, excreción fraccional de sodio, niños y adolescentes). Rev Chil Pediatr 2012; 83 (5): 438-444

\section{Introducción}

La obesidad es una enfermedad caracterizada por el aumento de la grasa corporal definida como tal en 1997 por la OMS ${ }^{1}$. Suele iniciarse en la infancia y adolescencia y se refiere a la existencia de un exceso acumulado de grasa corporal que se presenta como resultado de un balance positivo sostenido de energía, originado a su vez por un desequilibrio permanente entre la ingesta alimentaria y el gasto energético ${ }^{2,3}$.

La prevalencia de obesidad se ha incrementado en los últimos años considerándose una epidemia global y es la enfermedad crónica no transmisible más prevalente en el mundo contribuyendo a la elevada incidencia de diabetes, hipertensión, enfermedad cardiovascular y síndrome metabólico ${ }^{1,4-7}$. En la Argentina, la prevalencia de obesidad en población pediátrica oscila entre 4,1 y $11 \%$ según estudios realizados en la última década; estas cifras concuerdan con las descriptas en otras poblaciones a nivel mundial incluida América Latina ${ }^{1}$. La hipertensión arterial es una patología multifactorial subdiagnosticada en los niños, con tendencia a perpetuarse en la edad adulta, siendo el aumento de peso corporal un factor predictor de la misma ${ }^{5,8-11}$.

El riñón tiene un papel primordial en la regulación de la presión arterial, no sólo por su capacidad de modificar la excreción de agua, cloruro de sodio y otros electrolitos regulando el volumen circulante efectivo, sino por su complejo comportamiento como órgano endócrino productor de renina para la síntesis de angiotensina II, potente agente vasoconstrictor que estimula la producción de endotelina1 y potencia este efecto. Estas dos hormonas disminuyen la concentración de óxido nítrico (factor de relajación derivado del endotelio) aumentando la vasoconstricción, contrabalanceada por la acción vasodilatadora de prostaglandinas $\left(\mathrm{PGI}_{2}, \mathrm{PGE}_{2}\right)$ y bradicinina ${ }^{12-14}$.

Algunas evidencias han sugerido que altos contenidos de sal (cloruro de sodio) en la dieta a edades tempranas podrían generar elevación de la presión arterial o "programar" el desarrollo de hipertensión en el futuro en individuos genéticamente predispuestos, a partir de diversos mecanismos fisiopatológicos que junto a modificaciones neuroendócrinas generadas en la obesidad originan alteraciones de las funciones cardiovasculares y renales donde estaría alterada la excreción renal de sodio ${ }^{15-18}$.

Se considera que la excreción urinaria de sodio en $24 \mathrm{~h}$ es el patrón de oro ${ }^{19}$, para estimar la ingesta de sodio en población clínicamente sana, debido a que en estudios metabólicos se ha observado que el 93\% del aporte se elimina por orina y a que existen errores de los métodos basados en recordatorios de ingesta ${ }^{20}$.

El objetivo de este estudio fue evaluar el efecto de la obesidad sobre la excreción urinaria de sodio en niños y adolescentes.

\section{Pacientes y Método}

Estudio transversal que incluyó niños y adolescentes de ambos sexos, edades entre 5 y 15 años, que concurrieron al Servicio de Con- 
sultorio Externo del Hospital de Pediatría Dr. F. Barreyro, Posadas, Misiones, Argentina, en el período comprendido entre febrero de 2008 y diciembre de 2009. Se consideró como grupo control (C) a aquellos normopeso, definidos como un Índice de Masa Corporal (IMC) entre percentil 5 y 85 , que concurrieron al mencionado servicio, para obtener su correspondiente "certificado de buena salud", y como grupo de obesos (OB) a la población con IMC percentil $\geq 95$, que consultó por exceso de peso en dicho servicio durante el mismo período.

Se excluyeron aquellos con desórdenes endocrinos, antecedentes de enfermedad renal, trastornos genéticos, hipertensión, o con medicación que pudiera afectar el peso corporal, y/o la excreción de electrolitos.

El protocolo de estudio contó con la aprobación del Comité de Ética de dicho Hospital (13/12/2007). Todos los padres o tutores firmaron un formulario de consentimiento luego de la explicación del estudio y antes de su inicio, según los preceptos éticos de la declaración de Helsinki ${ }^{21}$.

Se midieron las siguientes variables antropométricas: peso, talla e índice de masa corporal (IMC).

El IMC se calculó como peso $(\mathrm{kg}) /$ talla $^{2}$ (m). Se definió a la población normopeso y obesa según IMC percentilo 5-85 y $\geq 95$ respectivamente $^{22}$. Peso: paciente de pie, con ropa interior y descalzo utilizando báscula de pie tipo CAM con resolución de $0,100 \mathrm{~kg}$. Talla: paciente de pie y descalzo utilizando cinta métrica adosada a la pared. El paciente fue medido en posición en plano de Frankfurt ${ }^{23}$.

La medición de la presión arterial fue realizada por un mismo operador, utilizando el método auscultatorio, en el brazo derecho con esfigmomanómetro aneroide y manguito adecuado al tamaño del brazo. Se consideró tensión arterial sistólica a la aparición del primer ruido de Korotkoff y tensión arterial diastólica a la desaparición de los ruidos (5to ruido de Korotkoff). Se definió hipertensión como el promedio de la tensión arterial sistólica o diastólica mayor o igual al percentilo 95 para la edad, sexo y talla medida en tres oportunidades distintas ${ }^{24,25}$.

Las muestras de sangre se obtuvieron lue- go de $12 \mathrm{~h}$ de ayuno. Todos los participantes del estudio recolectaron orina de $24 \mathrm{~h}$. Los participantes recibieron un recipiente para la recolección (lavado con detergente no iónico y enjuagado con agua destilada) y las siguientes instrucciones a sus padres: a una hora determinada de la mañana orinar y desechar, luego recolectar todas la muestras durante las próximas $24 \mathrm{~h}$ incluyendo la muestra del próximo día obtenida a la misma hora que comenzó la recolección.

Se realizaron las siguientes determinaciones: glucemia por método enzimático (GOD/ $\mathrm{POD})$ con colorimetría final según Trinder-CV $6,76 \%$ (Wiener) para excluir diabéticos, creatinina en suero $\left(\mathrm{Creat}_{\mathrm{s}}\right)$ y orina $\left(\mathrm{Creat}_{\mathrm{ur}}\right)$ por método colorimétrico-cinético-CV 8,5\%. (Boheringer) para realizar el cálculo del clearence de creatinina (DCE) como indicador de velocidad de filtración glomerular. Las lecturas fueron realizadas con espectrofotómetro BTS 320 (Biosystems). Las concentraciones de sodio sérico $\left(\mathrm{Na}_{\mathrm{s}}\right)$ y urinario $\left(\mathrm{Na}_{\mathrm{ur}}\right)$ se determinaron por electrodo ión selectivo (ISE), Easy Lyte Plus-Médica, CV 2,4\%.

El DCE fue calculado como: Creat ${ }_{u r} \times$ Diu-

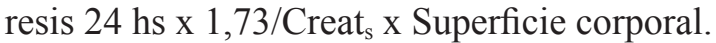

Se calculó la Excreción fraccional de sodio $(\mathrm{EFNa} \%)$ según la fórmula $\left(\left(\mathrm{Na}_{\mathrm{ur}} / \mathrm{Na}_{\mathrm{s}}\right) /\left(\mathrm{Creat}_{\mathrm{ur} /}\right.\right.$ Creat $\left.\left.{ }_{\mathrm{s}}\right)\right) \times 100$.

Para monitorear la calidad de todos los resultados se procesó como control interno Standatrol (Wiener), y como control externo muestras ciegas provenientes de la Fundación Bioquímica Argentina (Control de calidad PEEC).

La ingesta estimada de sal, utilizando la excreción urinaria de sodio en $24 \mathrm{~h}$ como marcador bioquímico, en una muestra de población pediátrica clínicamente sana, fue obtenida según el siguiente cálculo:

Ingesta de sal (mg/día): Sodio urinario $\left(\mathrm{Na}_{\text {ur }}\right) \mathrm{mmol} / \mathrm{dia} \times 58,5 \mathrm{mg} / \mathrm{mmol}$.

Se utilizó la prueba de Shapiro-Wilks para determinar la distribución de las variables cuantitativas. Para aquellas con distribución normal se usó el test t Student y se expresaron como media \pm desvío estándar. Para las variables que no presentaron distribución normal se empleó el test no paramétrico de Mann-Whitney y los datos se expresaron en mediana y 
rango. Se utilizó un nivel de significación estadística del 5\%. El análisis estadístico se llevó a cabo mediante el uso del programa Statgraphic 5.126,27.

\section{Resultados}

En el período comprendido entre febrero de 2008 y diciembre de 2009 se evaluaron 266 niños y adolescentes, edad promedio $10 \pm 3$ años. De acuerdo al estado nutricional $112(42 \%)$ eran normopeso y 154 (58\%) obesos. La distribución según el género fue: el sexo femenino del grupo control estuvo compuesto por $61(54 \%)$ y el de obesos por $85(55 \%)$ integrantes. El sexo masculino incluyó 51 (46\%) participantes controles y 69 (45\%) obesos.

La ingesta estimada de sal en el grupo normopeso fue 2,3 a 15,6 g de sal/día, obtenida a partir de los valores de excreción urinaria.

Los datos basales de los participantes se muestran en la tabla 1. Las presiones arteriales sistólica y diastólica fueron significativamente mayores en los niños obesos; no se encontraron diferencias significativas para la filtración glomerular.

El grupo control presentó concentraciones de sodio sérico, sodio urinario y $\mathrm{EFNa} \%$ dentro de los valores de referencia. Se observó un descenso significativo de la excreción urinaria de sodio y la $\mathrm{EFNa} \%$ en la población obesa. No se encontraron diferencias significativas en los valores de sodio sérico entre ambos grupos (tabla 2).

\section{Discusión}

El aporte principal de este estudio radica en la evaluación temprana de niños $\mathrm{y}$ adolescentes obesos, en los cuales estaría involucrado el manejo anormal de sodio, que podría generar hipertensión, de manera que se puedan tomar medidas orientadas a la prevención primaria. Este trabajo cobra relevancia ya que no se han
Tabla 1. Características basales de los participantes

\begin{tabular}{|lccc|}
\hline Variables & $\begin{array}{c}\text { C } \\
\mathbf{n}=\mathbf{1 1 2}\end{array}$ & $\begin{array}{c}\text { OB } \\
\mathbf{n}=\mathbf{1 5 4}\end{array}$ & $\mathbf{p}$ \\
\hline Edad (años) & $10 \pm 3$ & $10 \pm 3$ & 0,799 \\
Sexo (M/F) & $51 / 61$ & $69 / 85$ & \\
PAS (mmHg) & $98 \pm 10$ & $107 \pm 13$ & $<0,0001$ \\
PAD (mmHg) & $58 \pm 10$ & $64 \pm 12$ & 0,00004 \\
Glucosa (g/l) & $0,85 \pm 0,09$ & $0,85 \pm 0,10$ & 0,669 \\
\hline DCE (ml/min/SC) & $116 \pm 41$ & $123 \pm 47$ & 0,257 \\
\hline
\end{tabular}

C: controles, OB: obesos, PAS: presión arterial sistólica, PAD: presión arterial diastólica, DCE: clearence de creatinina, p: significancia estadística. t de Student

Tabla 2: Valores de Na sérico, excreción urinaria de Na y EFNa \% en la población estudiada

\begin{tabular}{|lccc|}
\hline Variable & $\begin{array}{c}\text { C } \\
\mathbf{n}=\mathbf{1 1 2}\end{array}$ & $\begin{array}{c}\text { OB } \\
\mathbf{n}=\mathbf{1 5 4}\end{array}$ & $\mathbf{p}$ \\
Na s(mEq/l) & 140 & 140 & 0,35 * \\
& $(136-147)$ & $(135-146)$ & \\
Na ur (mEq/kg/día) & 3,4 & 2,23 & $<0,0001^{* *}$ \\
& $(0,98-7,93)$ & $(0,34-6,26)$ & \\
EFNa\% & 0,71 & 0,59 & $0,0011^{* *}$ \\
& $(0,13-3,23)$ & $(0,17-1,85)$ & \\
\hline
\end{tabular}

C: controles, $\mathrm{OB}$ : obesos, $\mathrm{Na}_{\mathrm{s}}$ : sodio sérico, $\mathrm{Na}_{\mathrm{ur}}$ : sodio urinario, $\mathrm{EFNa}$ \%: excreción fraccional de sodio, p: significancia estadística . ${ }^{*}$ de Student. ${ }^{* *}$ Mann-Whitney

encontrando un gran número de investigaciones sobre las alteraciones en la homeostasis del sodio en obesidad infantil.

Si bien se excluyeron niños hipertensos para poder evaluar el efecto de la obesidad sobre la homeostasis del sodio, se observó que la población obesa presentó valores de presión arterial sistólica y presión arterial diastólica significativamente mayores que la población control, lo que podría contribuir, junto a otros factores, a generar hipertensión en la edad adulta.

En condiciones fisiológicas, aproximadamente el 98\% de la ingesta de cloruro de sodio se absorbe en el intestino delgado y se distribuye en el líquido extracelular, manteniéndose fuera de la célula por la acción de la ATPasa $\mathrm{Na} / \mathrm{K}$. El cloruro de sodio ingerido (aproximadamente 93\%) es excretado por el riñón, conservando el balance entre la ingesta y la excreción y regulando la presión arterial ${ }^{13,28}$. Esto ha permitido estimar la ingesta habitual de sal en 
la población normopeso utilizada como grupo control que presentó una ingesta habitual elevada de sal (2,3-15,6 g/día) mayor a sus necesidades fisiológicas y a las metas recomendadas (menor a 5,8 g/día).

Estos hallazgos son similares a los obtenidos en un estudio previo realizado por los autores ${ }^{29}$.

En los individuos obesos es incorrecto estimar la ingesta diaria de sal a través de la excreción urinaria de sodio porque presentan una alteración en el manejo renal de dicho ion; como esta población obesa proviene de las mismas zonas y concurre al mismo servicio que la población normopeso se consideró que su ingesta diaria de sal sería también elevada. Se podría estimar en estudios posteriores la ingesta de sal en individuos obesos eliminando de esta forma una variable de confusión, mediante un estudio de cohorte, con el consumo de sal controlado por el operador.

La disminución estadísticamente significativa de la excreción de sodio y excreción fraccional de sodio encontrada en la población estudiada, no sería ocasionada por una ingesta de sal disminuida que podría generar retención de dicho ion debido a mecanismos compensatorios del riñón, sino que se debería a la interrelación de los diversos mecanismos generados en la obesidad, a saber: a) activación del sistema nervioso simpático vasoconstrictor que estimula la reabsorción de sodio en los diversos segmentos de la nefrona a través de los siguientes transportes: simporte sodio/ soluto en túbulo proximal, simporte $\mathrm{Na} / \mathrm{K} / 2 \mathrm{Cl}$ $\left(\mathrm{NCCK}_{2}\right)$ en segmento ascendente grueso del asa de Henle, simporte $\mathrm{Na} / \mathrm{Cl}$ (NCC) en túbulo contorneado distal y canal epitelial de sodio $(\mathrm{ENaC})$ en túbulo conector-conducto colector $^{11,17}$; b) activación del sistema reninaangiotensina II-aldosterona: angiotensina II de acción vasoconstrictora, estimula el antiporte $\mathrm{Na} / \mathrm{H}\left(\mathrm{NHE}_{3}\right)$ en túbulo proximal mientras que aldosterona estimula tranportes de sodio fundamentalmente el canal epitelial de sodio en túbulo conector-colector aumentando ambas hormonas la reabsorción renal de sodio $^{5,10,12,13,18}$; c) hiperinsulinemia compensatoria a la insulinoresistencia que estimula la reabsorción de sodio en los distintos segmen- tos de la nefrona: túbulo proximal (antiporte $\mathrm{Na} / \mathrm{H})$ y canal epitelial de sodio en túbulo conector-conducto colector, además de generar disfunción endotelial con disminución de la producción de óxido nítrico ${ }^{30,31}$; d) hiperleptinemia con resistencia a la leptina que activa el sistema simpático, estimula reabsorción renal de sodio directa e indirectamente y genera disfunción endotelial con disminución de la producción de óxido nítrico ${ }^{15,32,33}$; e) disminución de la actividad biológica de los péptidos natriuréticos con descenso de la natriuresis ${ }^{10,18}$; f) disfunción endotelial con disminución del óxido nítrico vasodilatador y aumento de endotelina $_{1}$ vasoconstrictora $^{33} ; \mathrm{y}$ g) acumulación de calcio intracelular en músculo liso vascular que aumenta la vasoconstricción ${ }^{34}$.

Los resultados obtenidos en obesos coinciden con un estudio previo realizado por la University Medical School of Pecs en niños húngaros en 1996, quienes reportaron valores significativamente menores de excreción urinaria de sodio para la población obesa, haciendo hincapié en los mecanismos que se generan en la obesidad, para justificar dichas diferencias $^{30}$. Lurbe $\mathrm{E}$ y cols en el año 2000, encontraron una disminución en la excreción de sodio en niños obesos de la ciudad de Valencia (España) con respecto a niños no obesos, concluyendo que los obesos que poseen baja capacidad de excretar sodio, deben ser protegidos con una disminución de la ingesta de sal para prevenirlos de la hipertensión ${ }^{35}$. En otro estudio, Maldonado-Martín A y cols encontraron resultados compatibles con el presente estudio, sugiriendo que sus hallazgos son insuficientes para justificar un potencial beneficio en la restricción de sal en la dieta de los niños ${ }^{36}$. Se destaca que en los estudios citados previamente no se evalúa la ingesta estimada de sal para demostrar la retención de sodio en la población obesa.

\section{Conclusiones}

Los niños y adolescentes obesos, presentan alteración de la homeostasis del sodio con disminución de la excreción urinaria, teóricamente generada por la retención renal de dicho ion.

Estrategias efectivas para prevenir la obe- 
sidad podrían evitar el riesgo de desarrollo de hipertensión arterial.

\section{Referencias}

1.- Consenso sobre factores de riesgo de enfermedad cardiovascular en pediatría. Obesidad. Arch Argent Pediatr 2005; 103 (3): 262-81.

2.- Martínez Sopena MJ, Paz Redondo Del Rio M, Alonso Franch M: Valoración estado nutricional del obeso: estimación de la masa grasa. Bol Pediatr 2006; 46: 275-91.

3.- Roggiero E: Obesidad infantil. En: Torresani ME. Cuidado Nutricional pediátrico. Buenos Aires, Argentina: Editorial Universitaria de Buenos Aires 2001; 453-65.

4.- Hajer G, van Haeften TW, Visseren F: Adipose tissue dysfunction in obesity, diabetes, and vascular disease. Eur Heart J 2008; 29:2959-71.

5.- Bogaert YE, Linas $S$ : The role of obesity in the pathogenesis of hypertension. Nat Clin Pract Nephrol 2009; 5 (2): 101-11.

6.- Ebbeling C, Pawlak D, Ludwing D: Childhood obesity: public-health crisis, common sense cure. Lancet 2002; 360: 473-82.

7.- Filosof C, González C, Sereday M, Mazza C, et al: Obesity prevalence and trends in Latin-American countries. Obes Rev 2001; 2: 99-106.

8.- Sánchez Lozada LG, Franco M, Herrera Acosta J: Hipertensión arterial. Conceptos generales. En: García Nieto V, Santos Rodríguez F, Rodríguez Iturbe B. Nefrología Pediátrica. Madrid, España: Grupo Aula Médica 2006; 829-37.

9.- Saich Andonie C: Hipertensión arterial. En: Gordillo Paniagua G, Exeni RA, de la Cruz J. Nefrología Pediátrica. Barcelona, España: Elsevier España SA; 2003: 387-95.

10.- Aneja A, El-Atat FE, Mc Farlane SI, Sowers JR: Hypertension and Obesity. Recent Progr Horm Res 2004; 59 169-205.

11.- Hall JE, Hildebrandt DA, Kuo J: Obesity Hypertension: Role of Leptin and Sympathetic Nervous System. AJH 2001; 14: 103S-15S.

12.- Koeppen BM, Stanton BA: Transporte de agua y solutos a lo largo de la nefrona: función tubular. En: Koeppen BM, Stanton BA. Berne y Levy Fisiología. Madrid, España: Elsevier España SA; 2009: 578-93.

13.- Koeppen BM, Stanton BA: Control de la osmolalidad y el volumen de los líquidos corporales. En: Koeppen BM, Stanton BA. Berne y Levy Fisiología. Madrid, España: Elsevier España SA; 2009: 594-618.

14.- Rose BD, Post TW: Efectos de las hormonas sobre la función renal. En: Rose BD, Post TW. Trastornos de los electrolitos y del equilibrio ácido-base. Madrid, España: Marbán Libros SL; 2002: 163-238.

15.- Mathew B, Patel SB, Reams GP, Freeeman RH, Spear $R M$, Villarreal D: Obesity-Hypertension: Emerging Concepts in Pathophysiology and Treatment. Am J Med Sci 2007; 334 (1): 23-30.

16.- Wilson PW, D'Ágostino RB, Sullivan L, et al: Overweight and obesity as determinants of cardiovascular risk: The Framingham experience. Arch Intern Med 2002; 162: 1867-72.

17.- Esler M, Straznicky N, Eikelis N, et al: Mechanisms of Sympathetic Activation in Obesity- Related Hypertension. Hypertension 2006; 48: 787-96.

18.- Zhang R, Reisin E: Obesidad-hipertensión: efectos sobre los sistemas cardiovascular y renal. AJH 2001; 3: 156-6.

19.- De Courcy S, Michell H, Simmons D, Mac Gregor GA: Urinary sodium excretion in 4-6 year old children: a cause for concern? BMJ 1986; 292: 1428-9.

20.- Flaminio Fidanza F: Indicadores bioquímicos de la ingesta alimentaria. Rev Esp Nutr Comunitaria 2002; 8 (1-2): 46-50.

21.- Asociación Médica Mundial: Declaración de Helsinki. Principios éticos para las investigaciones médicas en seres humanos. http:/www.wma.net/es/30publications/ 10policies/b3/index.html.última visita: 01/11/2008.

22.- Centers for Disease Control and Prevention, Nacional Center for Health Statiscs CDC growth charts: EEUU 2000. Disponible en www.cdc.gov/nchs/about/ major/nhanes/growthcharts/charts.htm. última visita: 01/09/2007.

23.- Sociedad Argentina de Pediatría: Guías para la evaluación y crecimiento. $2^{\mathrm{a}}$. Ed. BsAs: Soc Arg Ped 2001; 100.

24.- Consenso sobre factores de riesgo de enfermedad cardiovascular en pediatría: Hipertensión arterial en el niño y el adolescente. Arch Arg Pediatr 2005; 103 (4): 348-65.

25.- The Fourth Report on the Diagnosis, Evaluation, and Treatment of High Blood Pressure in Children and Adolescents. Pediatrics 2004; 114 (2): 555-76.

26.- Pagano M, Gauvreau K: Comparación de dos medias. En: Pagano M., Gauvreau K. Fundamentos de Bioestadística México: Thomson Learning; 2001: 259-84.

27.- Pagano M, Gauvreau K: Métodos no paramétricos. En: Pagano M, Gauvreau K. Fundamentos de Bioestadística México: Thomson Learning; 2001: 302-22.

28.- Halperin ML, Goldstein MD: Sodium and water physiology. In Halperin ML, Goldstein MD. Fluid, electrolite, 
and acid-base- physiology. Philadelphia W.B.: Saunders Company; 1999: 227-82.

29.- Maskin de Jensen A, López M, Mir C, Martínez P: Sodio urinario como marcador bioquímico de la ingesta estimada de sal en niños y adolescentes. Acta Bioquím Clin Latinoam 2011; 45 (2): 279-85.

30.- Csabi G, Molnar D, Hartmann G: Urinary sodium excretion: association with hyperinsulinaemia, hypertension and sympathetic nervous system activity in obese and control children .Eur J Pediatric 1996; 155: 895-97.

31.- Shoko H, Seki G, Yamada H, Suzuki M, Koike K, Fujita $T$ : Insulin resistence, obesity, hipertension, and renal sodium transport. Internacional Journal of Hipertensión 2011; Article ID 391762, 1-8.

32.- Kshatriya S, Reams GP, Spear RM, et al: Obesity hypertension: the emerging role of leptin in renal and cardiovascular dyshomeostasis. Curr Opin Nephrol Hypertens 2010; 19: 72-8.

33.- Mark AL, Correia ML, Rahmouni K, Haynes WG: Selective leptin resistance: a new concept in leptin phisiology with cardiovascular implications. J Hypertens 2002; 20: 1245-50.

34.- Zemel MB: Nutritional and endocrine modulation of intracellular calcium: implications in obesity, insulin resistance and hypertension. Moll Cell Biochem 1998; 188: 129-36.

35.- Lurbe E, Álvarez V, Liao Y, et al: Obesity modifies the relationship between ambulatory blood pressure and natriuresis in children. Blood Press Monit 2000; 5 (5-6):
275-80.

36.- Maldonado Martin A, García Matarin L, Gil Extremera $B$, et al: Blood pressure and urinary excretion of electrolytes in Spanish schoolchildren. J Hum Hypertens 2002; 16 (7): 473-8.

37.- Polonia J, Maldonado J, Ramos R, et al: Estimation of salt intake by urinary sodium excretion in a Portuguese adult population and its relationships to arterial stiffness. Rev Port Cardiol 2006; 25 (9): 801-17.

38.- Maseko MJ, Majane HO, Milne J, Norton GR, Woodiwiss AJ: Salt intake in an urban, developing South African community. Cardiovasc J S Afr 2006; 17 (4): 186-91.

39.- Scientific Advisory Comittee on Nutrition, Salt and health. The Stattionery Office. London UKA 2003. Available from : http:/www.sacn.gov.uk/pdfs/sacn salt_final.pdf [consulta: 01/02/2007].

40.- Cooper R, Soltero I, Liu K, Berkson D, Levinson S, Stamler $J$ : The association between urinary sodium excretion and blood pressure in children. Circulation 1980; 32 (1): 97-104.

41.- McKeown NM, Day NE, Welch AA, et al: Use of biological markers to validate self-reported dietary intake in a random sample of the European Prospective Investigation into Cancer United Kingdom Norfolk cohort. Am J Clin Nutr 2001; 74 (2): 188-96.

42.- Byngham SA: The dietary assessment of individuals; methods accurancy, new techniques and recomendations. Nutr Abs Rev (Series A) 1987; 57: 705-42. 